\title{
¿Exclusión en los dos lados? Un análisis de las políticas de salud para migrantes mexicanos en Estados Unidos desde una perspectiva binacional
}

\section{Exclusion on Both Sides? An Analysis of Health Policies for Mexican Immigrants in the United States from a Binational Perspective}

\section{Exclusão nos dois lados? Análise das políticas de saúde para migrantes mexicanos nos Estados Unidos desde uma perspectiva binacional}

Fecha de recepción: 30-04-14 Fecha de aceptación: 09-05-14 Disponible en línea: 01-07-14 doi: 10.11144/Javeriana.rgyps13-27.edla

Cómo citar este artículo:

Sánchez-Siller IM, Gabarrot-Arenas, M. ¿Exclusión en los dos lados? Un análisis de las políticas de salud para migrantes mexicanos en Estados Unidos desde una perspectiva binacional. Rev. Gerenc. Polít. Salud. 2014; 13(27): 147-167. http://dx.doi.org/10.11144/Javeriana.rgyps13-27.edla

Estudiante del Doctorado en Ciencias Sociales del Tecnológico de Monterrey, Campus Monterrey, México. Correo electrónico: izasiller@gmail.com. Dirección de correspondencia: Cátedra Desarrollo Social y Globalización, Departamento de Relaciones Internacionales y Ciencia Política, Tecnológico de Monterrey, Ave. Eugenio Garza Sada 2501 Sur, Col. Tecnológico, CP 64849. Monterrey, Nuevo León, México.

** Profesora-investigadora y directora de la Cátedra sobre Desarrollo Social y Globalización del Departamento de Relaciones Internacionales y Ciencia Política del Tecnológico de Monterrey, Campus Monterrey, México. Correo electrónico: mariana.gabarrot@itesm.mx 


\section{Resumen}

Dentro del presente trabajo se analizó el acceso a los servicios de salud que tienen los migrantes mexicanos en Estados Unidos y en México. Mediante una revisión del marco legal e institucional, así como de la literatura del tema, se planteó un análisis del tipo de atención, acceso, derechos y restricciones que tienen las personas en ambos países. Lo anterior para argumentar que las políticas de salud actuales, enfocadas en la territorialidad de los derechos de salud, generan una doble exclusión para las poblaciones migrantes. Por un lado, la legislación migratoria en Estados Unidos deriva en un acceso cada vez más restrictivo; por otro, se ilustró cómo el sistema de salud mexicano no provee un acceso real debido a limitaciones en su estructura, recursos y diseño. Finalmente, se exploraron alternativas de acceso que tienen los migrantes dado el contexto actual, estableciendo algunas recomendaciones generales para una mejor inclusión en el futuro.

Palabras clave: salud; migración; servicios de salud; accesibilidad a los servicios de salud; política de salud; legislación sanitaria

\section{Abstract}

In this work we analyze the access to health services that Mexican immigrants have in the United States and Mexico. We bring up an analysis of the kind of attention, access, rights and restrictions that people have in both countries through a review of the legal and institutional framework, as well as the literature on the subject. This with the purpose of arguing that current health policies, focused on the territoriality of health rights, create a double exclusion for populations of immigrants. On the one hand, the United States migratory policies derive in a progressively more restrictive access; on the other, it is shown how the Mexican health system does not provide real access due to structural, resource, and design limitations. Finally, we explore access alternatives available to immigrants given the current context, establishing several general recommendations for a better inclusion in the future.

Keywords: health; migration; health services; accessibility to health services; health policy; health legislation

\section{Resumo}

Dentro do presente trabalho analisou-se o acesso aos serviços de saúde que tem os migrantes mexicanos nos Estados Unidos e no México. Mediante revisão do quadro legal e institucional, assim como da literatura sobre o tema, levantou-se uma análise do tipo de atendimento, acesso, direitos e restrições que tem as pessoas em ambos os países. Isso para argumentar que as atuais políticas de saúde, focadas na territorialidade dos direitos de saúde, geram uma dupla exclusão para as populações migrantes. Por um lado, a legislação migratória nos Estados Unidos deriva em um acesso cada vez mais restritivo; por outro, já foi mostrado como o sistema de saúde mexicano não fornece acesso real devido a limitações na sua estrutura, recursos e concepção. Finalmente, exploraram-se alternativas de acesso que tem os migrantes dado o contexto atual, estabelecendo algumas recomendações gerais para mais inclusão no futuro.

Palavras-chave: saúde; migração; serviços de saúde; acessibilidade aos serviços de saúde; política de saúde; legislação sanitária 


\section{Introducción: la salud en la agenda migratoria}

El acceso a la salud es uno de los indicadores básicos del desarrollo y ha sido un punto importante en las agendas de los derechos de los migrantes, a la par del derecho a la educación. A grandes rasgos, en la literatura sobre migración estos temas se han discutido en el marco de las definiciones de la ciudadanía $(1,2)$. Es decir, para determinar qué tipo de inmigrantes tienen acceso a qué tipo de servicios del Estado de Bienestar, se analiza principalmente su estatus legal y la adscripción formal de la nacionalidad. El resultado más evidente de estas discusiones es el hecho de que la mayor parte de las poblaciones móviles no tienen acceso a los servicios sociales, particularmente a los de salud, en los países de destino (3).

Sin embargo, es importante notar que en su mayoría, los migrantes provienen de comunidades de origen en donde también son excluidos de sus derechos, por vivir en condiciones de pobreza. Aunque es sabido que los migrantes no son los más pobres, sino quienes tienen acceso a los recursos para pagar los gastos de viaje e instalación, no deja de ser verdad que provienen mayormente de zonas empobrecidas del planeta. En su mayoría, los flujos tienen su origen en países donde no se ha logrado la cobertura apropiada de servicios básicos de salud y educación (4-7).

Por ello, la literatura aborda el tema principalmente desde dos ángulos: las investigaciones orientadas a las comunidades de destino y las orientadas a las comunidades de origen. El primer grupo se ha concentrado en problemáticas tales como el uso del sistema de salud en Estados Unidos y las alternativas que tienen los migrantes cuando son excluidos. Así, se ha mostrado que recurren a estrategias de remedios caseros y automedicación, consulta telefónica a familiares, atención en centros de beneficencia o caridad, consultas privadas o retorno a fronteras y comunidades de origen (8-12). También se han analizado las barreras para el acceso a la salud con base en el estatus legal, el desconocimiento del idioma inglés, el alto costo de los servicios, la discriminación y las largas esperas previas a las citas médicas. Otro aspecto importante es el análisis de las creencias relacionadas con el proceso de acceso, como lo son el miedo a la deportación y la ansiedad (13-15). En este sentido, se ha trabajado el tema de la salud psicofísica de los que migran y se ha demostrado que tienden a tener problemas para dormir, tristeza, sentimientos de soledad o nostalgia por la familia (16). Finalmente, existen estudios muy puntuales sobre la salud de los trabajadores agrícolas y su inseguridad alimentaria en zonas como California, Colorado y Carolina del Norte (17-23).

El segundo gran cuerpo de literatura constituido por los estudios referentes a las comunidades de origen - se puede dividir en cinco grandes temas: aquellos que se centran en el uso de hospitales en el lado mexicano de la frontera $(7,10,24,25)$; diagnósticos de la salud de los migrantes de retorno (26); enfermedades de transmisión sexual, principalmente VIH (27-30); salud reproductiva (14, $31,32)$ y salud infantil (33-35). En general, las investigaciones están enfocadas en un solo polo del flujo migratorio, por lo cual reflejan preocupaciones pertinentes principalmente al contexto inmediato.

Este artículo propone considerar un enfoque transnacional, para ir más allá de la unipolaridad. Si bien la definición de lo transnacional ha sido objeto de mucho debate académico, existe un consenso de que esta perspectiva ayuda a comprender el espacio social de los migrantes como un continuo, lo cual permite entender la migración y su impacto en ambos lados de la frontera (36- 
38). Es importante señalar que la literatura sobre el transnacionalismo se ha enfocado más bien a entender la construcción de espacios sociales transfronterizos, abordando temas como la identidad, la comunidad y el género (39-45). Si bien se ha trabajado en la conceptualización del desarrollo como un proceso transnacional, aún son pocos los trabajos que abordan temas específicos en materia de derechos sociales $(5,6,46-49)$. De tal forma, queda pendiente profundizar en el análisis de la integración de los migrantes en los países de destino, a la vez que se consideran las condiciones de acceso al bienestar en los lugares de origen.

De tal forma, la perspectiva transnacional hace posible entender la dinámica de doble exclusión que opera para estas poblaciones, particularmente cuando son consideradas como sujetos de derechos relacionados con la salud. Este trabajo tiene como objetivo general contribuir al entendimiento de este fenómeno, partiendo de un análisis del marco legal e institucional en el caso de la migración México-Estados Unidos. En primer lugar, se examinan los programas sociales vigentes y las legislaciones que restringen $\mathrm{u}$ otorgan derechos para el acceso a los servicios de salud. En Estados Unidos, estos presentan una clara evolución desde la década de los sesenta, con el surgimiento de los programas de la "Gran Sociedad" de Lyndon B. Johnson, hasta la Ley de Protección al Paciente y Cuidado de Salud Asequible promulgada por el actual presidente Barak Obama en el 2010. En México, inician con la consolidación del Estado de Bienestar en 1940 y culminan con la creación del Sistema de Protección Social en Salud en el 2002. Posteriormente, se lleva a cabo un análisis de las instancias institucionales, generadas por este marco regulatorio, a las cuales los ciudadanos mexicanos pueden acudir como migrantes en Estados Unidos y también como usuarios cuando están en México.

\section{Método}

En primera instancia se efectuó una revisión histórica de la legislación en materia de migración, salud y derechos sociales, identificando aquellos ordenamientos que de alguna forma confieran, modifiquen o restrinjan los derechos de los migrantes tanto en Estados Unidos como en México y que por tanto impacten en el acceso a los servicios de salud por parte de esta población. Asimismo, se realizó un análisis de los derechos conferidos en programas sociales e instancias institucionales en México y en Estados Unidos

Ahora bien, para una mejor comprensión de la evolución de los derechos al acceso a los servicios de salud por parte de la población migrante, consideramos necesario presentar una breve contextualización del caso particular de los mexicanos en Estados Unidos, por lo cual la siguiente sección presenta de forma breve las dimensiones del fenómeno migratorio entre ambos países, así como algunas precisiones conceptuales útiles para el análisis. La segunda y la tercera sección muestran los resultados del análisis del marco legislativo e institucional para determinar el grado de acceso a la salud en Estados Unidos y en México, respectivamente. Después se presenta una comparación, demostrando la tendencia hacia la exclusión en ambos lados de la frontera. Finalmente, se discuten las alternativas que los migrantes han desarrollado para contrarrestar la falta de acceso a los servicios de salud y se presenta una discusión de los limitantes detectados en la literatura a raíz de este análisis, para poder presentar algunos lineamientos generales de propuestas para el diseño de políticas y el estudio de las poblaciones migrantes desde un enfoque transnacional. 


\section{Resultados}

\section{El contexto: la migración México-Estados Unidos y el acceso a la salud}

Como es bien sabido, México tiene una larga tradición de migración a Estados Unidos, la cual a grandes rasgos inicia con la construcción del ferrocarril en ambos países y el auge de la agricultura en los Estados Unidos durante el siglo XIX. El flujo, dirigido principalmente hacia California y el sur de Texas, no ha sido interrumpido desde entonces. Lo anterior ha generado lo que se conoce como una tradición migratoria, especialmente en los estados de la zona oeste del país como Guanajuato, Jalisco, Michoacán, Durango, San Luis Potosí, Zacatecas, Nayarit y Colima. Sin embargo, a lo largo de los años, han ocurrido cambios importantes. Por un lado, el perfil de los migrantes ha ido cambiando, de trabajadores agrícolas temporales, predominantemente hombres, a empleados en el sector servicios, con una creciente participación femenina. Asimismo, la geografía se ha diversificado tanto en las regiones de origen (integrando estados como Oaxaca y San Luis Potosí) como en las de destino (con flujos hacia estados como Georgia y Oregon) $(50,51)$.

Según el Pew Hispanic Center, en el 2012 había un total de 33,4 millones de hispanos de origen mexicano en los Estados Unidos, de los cuales el $33 \%$ no tiene seguro de salud. Si tomamos en cuenta únicamente a la población nacida en México (11,4 millones), la proporción sin cobertura aumenta al $57 \%$. Lo anterior puede estar asociado al hecho de que un porcentaje similar $(51 \%)$ se encuentra en condición de indocumentados. Si bien las mediciones de poblaciones móviles son difíciles y los cálculos siempre se prestan a debate, podríamos asumir que cuando mínimo, la cifra de exclusión de la salud es de alrededor de seis millones de personas. Por otro lado, en México, de acuerdo con cifras del Consejo Nacional de Evaluación de la Política Social, 60,7\% del total de 112 millones de mexicanos carecían de seguridad social en el 2010, porcentaje que aumentó al 61,2\% en el 2012. Esta cifra es notablemente menor en la carencia por acceso a servicios de salud, ya que del 2010 al 2012 disminuyó de 29,2 a 21,5\%. Sin embargo, los criterios para considerar que una persona se encuentra en esta situación de carencia es únicamente, cuando: "no cuenta con adscripción o derecho a recibir servicios médicos de alguna institución que los presta incluyendo el Seguro Popular, las instituciones públicas de seguridad social o los servicios médicos privados" (52) y no un acceso disponible, aceptable y de calidad.

Evidentemente, estas cifras son una aproximación y varían de acuerdo con el contexto específico de cada estado, sobre todo si se toman en cuenta otras variables como el género y la edad. Sin embargo, nos muestran cómo uno de los desafíos más significativos es conseguir cobertura de servicios para la población mexicana, tanto en Estados Unidos como en México.

En ambos casos, la regulación del acceso a la salud ha ido evolucionando a lo largo de la historia. Mientras que en Estados Unidos se ha restringido cada vez más el acceso para los migrantes (tanto documentados como indocumentados), en México se ha ampliado la cobertura formal pero continúan los retos de infraestructura. A continuación se realizará una revisión de la legislación y los programas vigentes, así como de la literatura sobre el tema, y se planteará un análisis sobre el tipo de atención, el acceso, los derechos y las restricciones que tienen las personas en los dos lados de la frontera. 
Análisis del marco institucional y el acceso a los programas públicos de salud por parte de los migrantes mexicanos en Estados Unidos

Hasta la década de los sesenta, la provisión de los servicios de salud en los Estados Unidos estaba a cargo principalmente de los gobiernos de los estados. Durante esta época y debido al alto costo de la provisión de salud, todas las personas con bajos recursos, incluyendo a los migrantes, eran tratadas en los centros de salud sin fines de lucro, los cuales debían facilitar atención gratuita o de caridad $(53,54)$.

Sin embargo, en el gobierno del presidente Lyndon B. Johnson, el Congreso aprobó los llamados programas de la "Gran Sociedad", los cuales suponen un parteaguas en la provisión de salud, cambiando el enfoque desde la regulación y financiamiento estatal hacia lo federal y transformando el otorgamiento de servicios médicos de bajo costo o gratuitos de un "regalo paternalista", otorgado mediante caridad, a un derecho debidamente garantizado por el Estado (54).

En este sentido, la cobertura de los migrantes mexicanos pasa a depender en gran medida de estos amplios lineamientos legales, los cuales inician con una inclusión relativamente amplia para los migrantes, pero evolucionan hacia un régimen cada vez más restrictivo. A continuación se presentan estos cambios históricos, divididos en cuatro grandes etapas: 1) los mencionados programas insignia de los sesenta, 2) la regulación del acceso a salas de emergencia, la cual se dio durante los setenta y ochenta, 3) el incremento de las restricciones de los años noventa y, finalmente, 4) las últimas reformas iniciadas en el año 2000.

\section{Los programas de Gran Sociedad (1960-} 1970). Los programas generados en este 152 periodo son quizás los emblemáticos del sistema de salud estadounidense hasta el día de hoy: Medicare y Medicai (55). Fueron diseñados para llenar los vacíos existentes en el acceso a los servicios médicos para aquellas personas con bajos recursos o consideradas vulnerables por el Gobierno (niños, adultos mayores y discapacitados), que como se mencionó tenían atención médica mediante los centros de beneficencia.

El Medicare, quizás el programa más representativo de la provisión de salud de Estados Unidos, fue creado en 1965 bajo el título XVIII de la Ley de Seguridad Social (Social Security Act (SSA)). Su principal objetivo es otorgar seguro médico a personas de 65 años o mayores (sin importar sus ingresos o historial médico), personas con algún tipo de discapacidad y personas en fase terminal de alguna enfermedad renal. Un año después se creó el Medicaid como parte de las enmiendas a la Seguridad Social en donde se adicionó el título XIX a la sSA.

Medicaid provee atención médica a las personas de bajos recursos que no tienen seguro médico o que tienen un seguro médico no apropiado. El gobierno federal establece ciertos requisitos generales para la elegibilidad de los beneficios proporcionados por Medicaid; sin embargo, cada estado en particular determina los requisitos específicos para esta elegibilidad, así como el alcance de los servicios prestados.

La elegibilidad de estos programas se basaba en la finalidad de cada uno de ellos (adultos mayores, niños, personas con bajos recursos), pero el estatus legal era un requerimiento primordial. Por lo cual, mientras el migrante tuviera forma de comprobar que era residente legal (sin importar la ciudadanía) podía ser beneficiario de cualquiera de estos (56). Ahí se presenta la primera barrera en el sistema de acceso a servicios de salud mediante programas del gobierno: todos los 
migrantes que no contaban con documentos eran automáticamente inelegibles para estos.

Además, otro de los problemas que enfrentaban los migrantes durante estas épocas era la elegibilidad de los programas con base en el ingreso. Muchos de los migrantes que se encontraban trabajando legalmente en Estados Unidos no contaban con seguro médico laboral, ${ }^{1}$ ya que se concentraban en industrias que por lo general no ofrecen seguro de salud, tales como agricultura, limpieza y servicios de alimentación. Estos individuos carecían de los recursos suficientes para comprar un seguro privado y se les negaba la asistencia debido a que su ingreso era alto para calificar en los programas gubernamentales (57).

Después de la instauración de los programas de la Gran Sociedad, Estados Unidos comenzó con una serie de reformas tanto para regular el flujo migratorio como para establecer un uso normalizado de los hospitales (en especial sus salas de emergencia), periodo al cual le llamamos "de acceso a las salas de emergencia”.

\section{Periodo de acceso a las salas de emergencia} (1970-1980). En 1986 el Congreso de los Estados Unidos aprobó la Ley sobre el Tratamiento Médico de Emergencia y Parto Activo (Emergency Medical Treatment and Active Labor Act (EMTALA)), la cual daba acceso a los servicios de emergencia sin importar la capacidad del paciente de pagar sus cuentas; incluso migrantes indocumentados podían recibir atención médica cubiertos por Emergency Medicaid y cuidados obstétricos de Medicaid.

Debido a esta reforma y aunado al aumento de ingresos migratorios por la aprobación de la Ley de Reforma y Control de la Migración

1 Los empleadores no están obligados a otorgar seguro médico a sus empleados.
(Immigration Reform and Control Act (IRCA)), los centros de salud comenzaron a tener altos gastos que se solventaban mediante acuerdos para recibir donaciones voluntarias tanto de particulares como de empresas. Muchos de estos esquemas de financiamiento fueron eliminados a principios de los noventa; sin embargo, se creó la Ley de Reinversión y Recuperación (American Recovery and Reinvestment Act (ARRA)) en el 2009, que tiene como propósito proporcionar fondos a los centros de salud y así mitigar los múltiples costos por servicios a pacientes no asegurados (56).

La utilización de las salas de emergencia es una práctica común entre los migrantes, los cuales utilizan este servicio como una de las últimas alternativas y respaldados por el hecho de que no se les puede negar el servicio de emergencia. Según el estudio de Vargas Bustamante, realizado en el 2007 a través de la Encuesta de Salud en California (California Health Interview Survey (CHIS)), en los Estados Unidos se recibe un alto número de visitas de indocumentados a las salas de emergencia, que en su mayoría se tratan de casos de enfermedades agravadas por la evasión temprana de un tratamiento (58). Adicionalmente a este estudio, Akincingil demostró, mediante una muestra de migrantes que aplicaron a matricula consular en las ciudades con más migrantes mexicanos (Los Ángeles, Nueva York, Chicago, Atlanta, Dallas, Raleigh, Fresno) que los migrantes acudían a las salas de emergencia por enfermedades que se pudieron prevenir y que generalmente preferían esta opción por el miedo a la discriminación por parte de médicos, o simplemente porque no contaban con el dinero para pagarlo y la sala de emergencia es gratuita. Akincigil afirma que "cuando las políticas públicas dejan a los migrantes indocumentados con muy pocas opciones para la atención a la salud, ellos encuentran la sala de emergencia es su única opción” (13). 
Durante esta etapa, todos los migrantes legales eran considerados como cualquier ciudadano de Estados Unidos para efectos de los programas gubernamentales, no existía restricción alguna basada en su situación migratoria. Esto cambió en el periodo de restricción, en donde con la entrada en vigor de nueva legislación, se implementaron cambios sustanciales en los beneficios públicos. $\mathrm{Y}$ aunque esta reforma afectaba a todas las personas en Estados Unidos, por primera vez se hacía una distinción entre los migrantes ciudadanos y los no ciudadanos.

Periodo de restricción (1990-2000). En 1996 se aprobó la Ley General de Responsabilidad Personal y Oportunidad de Empleos (Personal Responsibility and Work Opportunity Reconciliation Act (PRWORA)). Esta "Reforma a la Seguridad Social" como es conocida, restringió el acceso a los programas públicos de beneficencia, basándose en el estatus migratorio, afectando ahora a los migrantes legales que tenían menos de cinco años en el país.

Para efectos de elegibilidad, PRWORA estableció dos categorías de inmigrantes: migrantes calificados y migrantes no calificados. Los migrantes calificados incluían: residentes legales permanentes (LPR en su sigla en inglés), refugiados, asilados y personas con otros estatus migratorios (como lo son niños y esposas de LPR). Los migrantes no calificados englobaban a los migrantes indocumentados, extranjeros y algunos migrantes legales (como estudiantes o turistas). Sin embargo, para los migrantes calificados la elegibilidad es efectiva a los cinco años después de obtener este título ${ }^{2,3}(55,59)$.

2 Únicamente aplica si la fecha de arribo del migrante fue después del 22 de agosto de 1996.

3 PRWORA estableció también excepciones de esta prohibición de los cinco años para ciertos grupos de migrantes (en adelante "las excepciones") como lo son: refugiados, asilados y otros migrantes bajo causas humanitarias, migrantes con cuarenta crédi-
Esta reforma afectó negativamente a la obtención de atención médica entre los migrantes, tanto por las nuevas restricciones, las crecientes condiciones para trabajar (las cuales contaban con menos posibilidades de proporcionar algún tipo de seguro médico), como por el temor creciente en los migrantes para acercarse a centros de salud o a su inscripción a programas. Por ejemplo, el estudio de Kaushal asegura que posteriormente a PRWORA el número de mujeres sin seguro médico, solteras, con poca educación y nacidas en el extranjero aumentó entre 9,9 y 10,7 puntos porcentuales. Del mismo modo, la proporción de niños sin seguro médico, nacidos en el extranjero creció 13,5 puntos porcentuales (60). Esto muestra que a pesar de que la reforma solo era aplicable para los "nuevos" migrantes, también tuvo repercusiones con las personas que anteriormente ya eran beneficiarias de algún programa de salud.

PRWORA, de igual forma, otorgó poder a los estados en dos formas: en primer lugar, el poder de instituir beneficios exclusivos de los estados con fondos de estos para los migrantes calificados durante la restricción de los cinco años, esto para reemplazar la pérdida de los beneficios federales, e incluso les dio la facultad de otorgar beneficios a migrantes no calificados. En segundo lugar, los estados pueden determinar requisitos adicionales a migrantes calificados para la elegibilidad de Medicaid (56).

Ante esta disposición algunos estados crearon programas para asegurar la atención médica para los residentes. Por ejemplo, Connecticut estableció un programa independiente, financiado por el estado para los migrantes legales. Por otro lado, Washington otorgó a los migrantes la posibilidad de pertenecer a un programa parecido, pero con beneficios limitados y costos compartidos (61).

tos de trabajo (qualifying quarters) (únicamente para SNAP), miembros de la militar, soldados veteranos (y sus esposas e hijos). 
En 1997 se creó el Programa de Seguro Médico para Niños (Children's Health Insurance Program (CHIP)), el cual es considerado el programa más grande de seguros médicos, después de la creación de Medicaid en los sesenta, y se encuentra bajo el título XXI de la SSA. Es un programa administrado por el Departamento de Salud y Servicios Sociales de los Estados Unidos que provee fondos cada estado para proporcionar seguros de salud a familias con niños. Este programa fue diseñado para cubrir a niños que no se encuentran asegurados y que viven en familias con ingresos bajos, pero no lo suficiente como para calificar para el programa Medicaid (56).

Uno de los mayores problemas en el acceso al programa CHIP, según Dordeski (61) se basa en el criterio de elegibilidad en la relación padre-hijo. Para efectos de la Ley de Seguridad Social, el niño es definido como un "hijo biológico, hijo adoptivo o hijastro dependiente" (62) o dependiente directo de sus abuelos y debe de haber una conexión reconocida legalmente. Ahora bien, en algunos casos de familias migrantes, los niños son criados por personas distintas a los padres o abuelos biológicos (como tíos, primos o incluso hermanos). Esto crea un problema al momento en que el adulto intenta solicitar beneficios para el niño, ya que siendo él quien está a cargo del menor, no puede satisfacer la definición de relación requerida por el sistema (61).

Periodo de reformas (2000-a la fecha). Finalmente, en el 2009 la Ley Federal de Reautorización del Programa de Seguro Médico para Niños (Children's Health Insurance Program Reauthorization Act (CHIPRA)) permitió a los estados cubrir a mujeres embarazadas y a niños calificados, sin tener en cuenta la restricción de los cinco años bajo Medicaid y/o chIP. A través de esta nueva ley, se proporcionó a los estados nuevos fondos y diversos incentivos con el objeto de apoyar a cada uno en el desarrollo de estrategias eficientes y eficaces para identificar, inscribir y retener la cobertura de salud para los niños y madres embarazadas que no contaban con algún seguro. Además, se proporcionó la flexibilidad para que los estados ampliaran la cobertura de atención para aquellos que lo necesitaran (55).

De acuerdo con lo dicho, en la tabla 1 se establecen los derechos y las restricciones para los migrantes a través de cada una de las etapas legislativas y en cada uno de los programas sociales otorgados por el Estado.

TABLA 1. DERECHOS Y RESTRICCIONES A TRAVÉS DEL TIEMPO

\begin{tabular}{|c|c|c|c|c|}
\hline Etapa & $\begin{array}{c}\text { Gran Sociedad } \\
(1960-1970)\end{array}$ & $\begin{array}{c}\text { Acceso a } \\
\text { emergencias } \\
(\mathbf{1 9 7 0 - 1 9 8 0 )}\end{array}$ & $\begin{array}{l}\text { Restricciones } \\
\text { (1990) }\end{array}$ & $\begin{array}{l}\text { Reformas } \\
(2000)\end{array}$ \\
\hline $\begin{array}{c}\text { Tipo de } \\
\text { acceso a los } \\
\text { migrantes }\end{array}$ & $\begin{array}{l}\text { - Se crearon como } \\
\text { parte de los pro- } \\
\text { gramas de la "Gran } \\
\text { Sociedad". } \\
\text { - Cualquier migran- } \\
\text { te documentado } \\
\text { podía ser elegible } \\
\text { para un programa } \\
\text { social. }\end{array}$ & $\begin{array}{c}\text { Cualquier persona } \\
\text { puede recibir ser- } \\
\text { vicio de Emergency } \\
\text { Medicaid. }\end{array}$ & $\begin{array}{c}\text { - Se restringe el } \\
\text { acceso a migrantes } \\
\text { legales con una } \\
\text { estancia menor a } \\
\text { cinco años. } \\
\text { - Le otorga el } \\
\text { poder a los estados } \\
\text { para decidir sobre } \\
\text { elegibilidad y bene- } \\
\text { ficios. }\end{array}$ & $\begin{array}{c}\text { CHIPRA } \\
\text { otorga facultad a } \\
\text { los estados de cu- } \\
\text { brir a mujeres em- } \\
\text { barazadas y niños, } \\
\text { sin tener en cuenta } \\
\text { la restricción de los } \\
\text { cinco años. }\end{array}$ \\
\hline
\end{tabular}

Los migrantes indocumentados no tienen acceso a los programas públicos de salud desde su creación. 
Ante este escenario de crecientes restricciones, se ha comprobado que algunos migrantes deciden regresar a territorio mexicano. Según Nigeda, esto puede ser: 1) a territorio fronterizo, debido al costo elevado en Estados Unidos y la facilidad de la cercanía para un posible regreso; o 2) al lugar de origen, cuando ya existe una incapacidad para trabajar o de costo elevado; 3) durante las visitas cortas, principalmente para acciones de prevención, tratamientos y control de problemas crónicos (10). Existen investigaciones que demuestran que los migrantes tienden a regresar más enfermos (o menos sanos) de lo que se fueron, como por ejemplo la de Ullmann, quien con base en el Proyecto de Migración Mexicana (Mexican Migration Program (MMP) demostró que los migrantes de retorno tienden a mostrar mayor prevalencia en enfermedades del corazón, desórdenes emocionales/psiquiátricos, obesidad y adicción al tabaco que los adultos no migrantes (3).

En la misma línea, el estudio de GonzálezBlock (24) demuestra que la utilización de hospitales por parte de los migrantes es distinta en dichas regiones. Mientras que en la frontera se asiste principalmente los servicios privados, en las comunidades de origen se acude a hospitales públicos. Por lo contrario, González-Block comenta que la decisión de regresar al país de origen no es muy frecuente en gente con más de 65 años o en maternidad, pues tienden a tener más protección legal en Estados Unidos. Sin embargo, el acceso en México también es más restringido de lo que parece, tal como se discute en la siguiente sección.

\section{Análisis del marco institucional y programas} públicos para el acceso a la salud en México

El sistema de salud en México, caracterizado

por su alto grado de segmentación (63), está conformado por dos sectores principales: el público y el privado. El público comprende la seguridad social (Instituto Mexicano del Seguro Social (IMSs) e Instituto de Seguridad y Servicios Sociales de los Trabajadores del Estado (ISSSTE), principalmente) y el proporcionado por el Gobierno a través de instituciones y programas que prestan servicios a la población sin seguridad social, principalmente el Seguro Popular. A continuación se describen brevemente cada una de las opciones de acceso divididas en dos grandes etapas: 1) el periodo de Estado de Bienestar y 2) y el periodo de reformas y programas sociales. De igual forma, se evalúa la factibilidad de que los mexicanos migrantes puedan acudir a ellas.

Estado de Bienestar (1940-1980). En México los servicios de seguridad social están amparados en el artículo 123 de la Constitución Política y a lo largo de la Ley Federal del Trabajo. Este sistema de seguridad social incluye instituciones federales, estatales, empresas paraestatales y otros organismos sociales. Aunque existen diversos proveedores de estos servicios de salud, como el Instituto de Seguridad Social de las Fuerzas Armadas (Issfam), Petróleos Mexicanos (Pemex), Luz y Fuerza del Centro (LFC) y la Comisión Federal de Electricidad (CFE), este servicio descansa principalmente en dos grandes instituciones: el Instituto Mexicano del Seguro Social (IMSs) y el Instituto de Seguridad Social y Servicios Sociales de los Trabajadores del Estado (ISSSTE), creados en 1942 y 1960 , respectivamente $(64,65)$.

Las instituciones de seguridad social en México son instancias paraestatales desconcentras, las cuales cuentan con instalaciones propias y recursos humanos propios (63). En la actualidad un poco más del $40 \%$ de la población es derechohabiente de seguridad social en México, sumando una cantidad de 42584535 personas (Instituto Nacional de Estadística y Geografía (Inegi), 2010). 
Cabe mencionar que existen dos formas de afiliación al IMss: por el régimen obligatorio y por el régimen voluntario. En el régimen obligatorio se encuentran los trabajadores, los miembros de sociedades cooperativas de producción y las personas que determine el Ejecutivo Federal a través del decreto respectivo. En el régimen voluntario pueden asegurarse los trabajadores independientes, los trabajadores en industrias familiares, los empleadores y los trabajadores al servicio de las administraciones públicas de la Federación, entidades federativas y municipios que no cuenten con otra cobertura de la seguridad social (66).

Adicionalmente, en 1979 el Gobierno extendió la cobertura de los servicios de salud a las zonas rurales más "apartadas, aisladas y dispersas" las cuales carecían de servicios básicos de salud, mediante un convenio entre el IMss y la Coordinación General del Plan Nacional de Zonas Deprimidas y Grupos Marginados, a través del cual se creó el IMSsCoplamar (ahora conocido como el IMSsOportunidades). Este programa se encarga de extender los beneficios de la seguridad social a grupos marginados de México. Los servicios son abiertos a la población, es decir, cualquier persona recibe la prestación del servicio en forma completamente gratuita y para ello se solicita únicamente la inscripción al Padrón de Beneficiarios mediante la entrega de los siguientes documentos: clave única de registro de población (CURP) y comprobante de domicilio. Los beneficiarios de este programa pueden también estar afiliados a algún sistema de aseguramiento, únicamente tendrán que presentar un comprobante como: la póliza de afiliación al Seguro Popular o al Programa Desarrollo Humano Oportunidades, afiliación al IMSs, Issste, Pemex, Secretaría de la Defensa Nacional (Sedena) o Secretaría de MarinaArmada de México (Semar), o afiliación a los Sistemas Estatales de Seguridad Social (67).
Actualmente, los servicios de atención médica de Imss-Oportunidades se dan en unidades médicas rurales y hospitales rurales de los estados de Baja California, Campeche, Coahuila, Chiapas, Chihuahua, Durango, Hidalgo, Michoacán, Nayarit, Oaxaca, Puebla, San Luis Potosí, Sinaloa, Tamaulipas, Veracruz, Yucatán, Zacatecas, Guerrero y el estado de México. Estos servicios se dan en dos niveles de atención, el primero se concentra en los centros de salud con atención a servicios primarios y preventivos como diarreas, enfermedades respiratorias, nutrición, prevención y control de hipertensión, accidentes, entre otros. El segundo nivel de atención se concentra en los hospitales rurales con atención continua e integral de consulta externa en medicina de especialidades. Para las personas que requieren atención de tercer nivel, por la complejidad del padecimiento, se canaliza a hospitales de la Secretaría de Salud (68).

En general, la atención a la salud a través de la seguridad social (IMSs), a pesar de ser una de las principales formas de obtener estos servicios en México y de las más completas, no es una alternativa para las personas que migran, toda vez que al momento de dejar de trabajar y de realizar las debidas aportaciones en el país, pierden el derecho de recibir los beneficios otorgados por cualquiera de las instituciones mencionadas y por lo tanto los integrantes de su familia. En consecuencia, tienen que recurrir a los servicios proporcionados por el Estado para la población que no es derechohabiente de seguridad social o a la atención privada.

\section{Periodo de reformas y programas sociales} (1980-a la fecha). Este periodo de reformas comienza en 1982 con la adición del artículo $4^{\circ}$ de la Constitución mexicana, el cual eleva a rango constitucional el derecho a la protección a la salud. A partir de este momento comienza la aplicación de programas sociales 
mediante los cuales se proporcionan servicios de salud a los cuales pueden acudir tanto a los migrantes como sus familias.

Por otro lado, en el año del 2002 se adicionó el título $3^{\circ}$ Bis a la Ley General de Salud, el cual dio lugar a la creación del Sistema de Protección Social en Salud (SPSs), toda vez que el artículo 77 Bis-1 establece que todos los ciudadanos de México tienen derecho a ser incorporados al Sistema de Protección Social en Salud de conformidad con el artículo $4^{\circ}$ de la Constitución, sin importar su condición social. Este derecho consiste básicamente en que el Estado garantizará el acceso sin desembolso ni discriminación al momento de utilizar los servicios médicoquirúrgicos, farmacéuticos y hospitalarios en las necesidades de salud. Este servicio será como mínimo la atención en el primer nivel, así como la consulta externa y la atención en un segundo nivel (69).

El brazo operativo del spss es el Seguro Popular, el cual ofrece por primera vez en la historia del país acceso igualitario de un aseguramiento médico público a la población no asalariada. Básicamente, consiste en un sistema que consolida los recursos económicos, provenientes de diversas fuentes, ${ }^{4} \mathrm{a}$ fin de financiar el costo de los servicios de salud para la población que lo requiere. Esto significa que, a través del Seguro Popular, el Estado mexicano garantiza que la población reciba atención médica en los tres niveles, con sus restricciones (70). En primer lugar, el servicio de atención y los medicamentos otorgados son los establecidos dentro del marco del Catálogo Universal de Servicios de Salud (Causes). Por otra parte, mediante el manejo de un Fondo de Protección contra Gastos Catastróficos (FPGC), el cual cubre 49 diferentes acciones para atender en 17

4 Recursos de gobierno federal, entidades federativas y contribuciones de los afiliados. padecimientos como: cáncer cervicouterino, cánceres de niños y adolescentes, trasplante de médula, cuidados intensivos neonatales, trastornos quirúrgicos congénitos y adquiridos, cataratas y gastos por VIH/SIDA (67). Este fondo busca evitar el empobrecimiento por razones de enfermedad; sin embargo, Santos-Padrón asevera que la creación de este tiene debilidades, ya que además de ser muy limitado en comparación con la cobertura de la seguridad social, se creó mediante una consideración estática de la demanda sanitaria a través del tiempo y no precisa las necesidad reales de salud de la población (25).

Actualmente en todo México 20544362 familias, que suman un total de 52738355 personas, son beneficiarias de este programa y tienen acceso a servicios médico-quirúrgicos, farmacéuticos y hospitalarios. Sin embargo, la falta de infraestructura, de medicamentos y las malas condiciones de los centros de salud, crean barreras que dificultan la atención médica adecuada las personas beneficiarias de este programa (71).

En la tabla 2 se comparan el criterio de afiliación, el beneficiario y los beneficios de cada uno de los sectores mencionados, para así poder observar más claramente a cuál tipo de servicio tiene oportunidad un migrante de asistir.

Por lo anterior, el Seguro Popular se vuelve una de las opciones viables para las personas que migran, junto con la atención privada, ya que se otorga de forma individual y no requiere que toda la familia se encuentre en el mismo lugar, sino únicamente que sus miembros cumplan con los requisitos para ser beneficiarios del programa, y pueden gozar de estos servicios si son la cabeza de familia o un dependiente económico, o si fueron afiliados al momento de requerir asistencia médica (24). 
TABLA 2. Sectores para el aCCeso a servicios de Salud en México

\begin{tabular}{|l|l|l|l|}
\hline Sector & Criterio de afiliación & Beneficiario & Beneficios \\
\hline $\begin{array}{l}\text { Seguridad } \\
\text { Social }\end{array}$ & $\begin{array}{l}\text { Obligatoria para } \\
\text { empleados (existe el } \\
\text { régimen voluntario), } \\
\text { ser asalariado o fami- } \\
\text { liar, ser pensionado }\end{array}$ & $\begin{array}{l}\text { Empleado y } \\
\text { familiares }\end{array}$ & $\begin{array}{l}\text { Servicios de salud, } \\
\text { cobertura total de servicios médi- } \\
\text { cos y préstamos monetarios }\end{array}$ \\
\hline $\begin{array}{l}\text { Seguro } \\
\text { Popular }\end{array}$ & $\begin{array}{l}\text { Voluntaria, no tener } \\
\text { seguridad social, } \\
\text { pagar cuota familiar, } \\
\text { renovación periódica }\end{array}$ & $\begin{array}{l}\text { Titular inscri- } \\
\text { to y familiares }\end{array}$ & $\begin{array}{l}\text { Satúlogo Universal de Servicios de } \\
\text { Saluses), } \\
\text { servicios incluidos en el Fondo de } \\
\text { Protección contra Gastos Catas- } \\
\text { tróficos (FPG) }\end{array}$ \\
\hline $\begin{array}{l}\text { Atención } \\
\text { privada }\end{array}$ & N/A & $\begin{array}{l}\text { Población } \\
\text { con capacidad } \\
\text { económica }\end{array}$ & $\begin{array}{l}\text { Se paga por servicios y medica- } \\
\text { mentos requeridos }\end{array}$ \\
\hline
\end{tabular}

Fuente: elaboración propia

Sin embargo, no existen datos estadísticos certeros que proporcionen el número de migrantes que están inscritos en el Seguro Popular, además que como sostiene González-Block, generalmente es esta población la que pertenece al "pequeño grupo" de personas que no se afilian por falta de interés, basándose en los limitados esfuerzos realizados por parte de los consulados mexicanos en Estados Unidos para preafiliar a mexicanos, la cual ha tenido una respuesta pobre $(24,72)$.

Junto a esto, uno de los requisitos establecidos por el Seguro Popular para su afiliación es la presentación de dos documentos oficiales: el CURP y la credencial para votar (IFE), ${ }^{5}$ o documentos válidos que los sustituyan. ${ }^{6}$

5 La credencial para votar (IFE) es exclusivo para la persona que será el titular.

6 a) CURP: acta de nacimiento, certificado de nacimiento, carta de no registro o inexistencia o algún documento que contenga la clave CURP como: cédula de identificación fiscal, pasaporte, cédula profesional, cartilla de servicio militar; b) credencial para votar (IFE): pasaporte, licencia de
Esto representa un obstáculo tanto para los migrantes que no cuentan con documentos oficiales mexicanos a causa de su larga o circular estadía en Estados Unidos como para las familias llamadas de "estatus mixto". Estas familias tienen hijos que nacieron en Estados Unidos y a su retorno no cuentan con la documentación necesaria para su afiliación en el Seguro Popular como el resto de los integrantes mexicanos, estableciéndose con ello una clara exclusión, incluso dentro de la misma familia.

Aunado a esto se encuentra el factor de la restringida cobertura del Seguro Popular, la cual cubre únicamente ciertas enfermedades, a diferencia de la seguridad social, la cual tiene una cobertura amplia por cualquier enfermedad $(24,25,73)$. Además, GonzálezBlock (24) en su estudio sobre el uso de hospitales entre migrantes demostró que existen condiciones y tratamientos comunes

conducir, cartilla del servicio militar, matrícula consular, tarjeta INAPAM, carta de autoridad local, tarjeta de residencia. 
entre este grupo de población como lo es la diálisis, la cual es poco accesible debido al poco control de hipertensos y diabéticos en México (74) y representa un tratamiento de gran demanda entre los repatriados no cubierta por el Seguro Popular.

Ahora bien, la segunda y última opción que tienen los migrantes es la asistencia médica en servicios privados, la cual incluye no solo la consulta, sino también el medicamento, el tratamiento, el transporte, el material y en casos de mayor atención la hospitalización. Todo estos gastos implican un desembolso importante tanto en el individuo como en una familia, pero al final de cuentas es la alternativa más acudida por los migrantes y sus familias (24).

La pregunta ahora es, basándose en el interés y la capacidad de los migrantes mexicanos por afiliarse al Seguro Popular, estando en México o en Estados Unidos y los costos elevados de la seguridad privada, ise encuentran en un riesgo latente de quedar fuera de los seguros de salud en ambos países?

Desde el 2001 en México se creó el programa "Vete Sano y Regresa Sano". El cual consiste en un conjunto de acciones orientadas a informar, capacitar, difundir y comunicar a la población migrante en las comunidades de origen, traslado y destino, acerca de medidas de salud, red se servicios en las rutas de migración, albergues agrícolas, realización anual de la Semana de Salud, atención en ventanillas de salud en Estados Unidos, creación de la Tarjeta Binacional para el control de enfermedades y capacitación sobre temas de salud para el autocuidado. ${ }^{7}$ No obstante, la información de este programa otorgada a través de los medios oficiales, con respecto a su efectividad, o datos estadísticos que muestren su funcionamiento, es casi nula. Incluso Vizcarra-Bordi y Farfán-González (75) califican al programa como propagandístico, pues consideran que se dedica a difundir información sobre él mismo a través de discursos, programas en medios audiovisuales, etc. En su investigación los autores encontraron que dicho programa no funcionaba en zonas rurales del estado de México con alto índice migratorio, en donde ni siquiera se tenía conocimiento de su existencia.

A su vez en el 2001, instituciones del gobierno federal, estatal, locales, organizaciones comunitarias y voluntarios unieron esfuerzos para crear la Semana Binacional de Salud (SBS). Esta consiste en movilización comunitaria que tiene como fin mejorar la salud de la población latinoamericana migrante en Estados Unidos y Canadá. Dichas actividades son una reunión anual en el mes de octubre para realizar actividades como talleres educativos, canalización a servicios de salud y a programas de seguro médico, vacunas y exámenes básicos de detección gratuitos. Todas estas diligencias se realizar en coordinación con las ventanillas de salud de cada una de las representaciones consulares de México en Estados Unidos, en cooperación con agencias fiscales, organizaciones de la sociedad civil (OSC), gobiernos municipales y locales, universidades, hospitales públicos y centros comunitarios de los Estados Unidos. ${ }^{8}$ Según Farfán-González y Vizcarra-Bordi (76), este programa es el que mejor expansión ha tenido, ya que se enfoca en el estado de California y a la fecha abarca todo Estados Unidos, México, Canadá y algunos países de Centroamérica.

7 Para mayor información sobre el Programa se puede consultar su página web en la Secretaría de Salud (www.promocion.salud.gob.mx)

8 Ver: http://www.semanabinacionalmexicousa.org 
Enfoque comparativo y alternativas actuales de acceso a la salud - Estados UnidosMéxico

A lo largo del documento se ha analizado el marco institucional tanto de México como en Estados Unidos para entender la dinámica de doble exclusión en la cual se encuentran los migrantes. Por un lado, la falta de acceso a servicios de salud en el país de destino a causa del estatus migratorio en el que se encuentran, y por el otro, excluidos de sus derechos por las coberturas inapropiadas de los servicios básicos de salud y las condiciones de pobreza de sus comunidades de origen.

Por una parte, en Estados Unidos, siendo un migrante indocumentado o legal con una estancia menor a cinco años, es difícil ser elegible para algún programa de salud federal y esto se debe a la evolución legislativa que se vuelve cada vez más restrictiva, como se mencionó en la segunda parte del documento. Lo anterior puede variar dependiendo del estado en donde se resida; por ejemplo, en estados como California o Nuevo México se puede ser elegible sin importar la restricción. Sin embargo, existen otros estados como Arizona o Illinois en donde se excluye a todo migrante indocumentado o documentado en el tiempo de restricción (incluyendo niños y mujeres embarazadas). Por lo tanto, al tener un acceso restringido o nulo a los programas de salud en Estados Unidos, los migrantes se ven en la necesidad de recurrir a otras alternativas en el caso de alguna enfermedad.

Una de las opciones con que cuentan los migrantes es la atención en centros de beneficencia o de caridad que brinden servicios de salud. Los principales centros de atención de esta índole son los centros de salud calificados por el Gobierno Federal (federally qualified health centers (FOHC)) y los centros de salud de migrantes, los cuales son organizaciones sin fines de lucro financiadas por la Administración de Recursos y Servicios de Salud Federal (Health Resources and Services Administration (HRSA)), los cuales ofrecen atención primaria a poblaciones vulnerables como la de los migrantes, sin importar el estatus legal, la capacidad de pago o la situación con el seguro. La diferencia entre estos dos institutos es que los centros de salud de migrantes son únicamente para los trabajadores agrícolas temporales y sus familias $(9,12)$.

De acuerdo con el Consejo Nacional de Población, el 32\% de los migrantes mexicanos en Estados Unidos (documentados) obtienen los servicios de salud a través de los seguros privados o mediante consultas privadas externas, lo cual representa un alto gasto en el cual se tiene que incurrir en caso de enfermedad, dados los elevados costos en materia de salud en dicho país. Lo anterior se ve reflejado en la frecuencia con que los migrantes acuden a un servicio médico (de cualquier índole), ya que esta es cinco veces menor que la recurrencia de un ciudadano estadounidense. Cuando un migrante recurre a un servicio privado, por lo regular lo hacen con la esperanza que "el doctor no los delate" y por recomendación de algún otro migrante (10).

Finalmente, existen otras posibilidades en caso de enfermedad, como lo explica Nigeda (10) en su estudio realizado a migrantes en cuatro entidades de alta expulsión migratoria (Guanajuato, Michoacán, San Luis Potosí, Zacatecas e Hidalgo), en donde muestra las alternativas que los migrantes tomaban para la atención médica. En un primer escenario dentro de Estados Unidos, los migrantes recurrían a los siguientes medios: 1 ) remedios caseros o automedicación, el cual se daba en caso de patologías no graves y con consulta a familiares en México; 2) consulta telefónica a familiares, mediante las cuales los familiares van con un médico en la comunidad de ori- 
gen y se envían los medicamentos. Es importante señalar que esta consulta y compra de medicamento se realiza en establecimientos privados (consultorios y farmacias).

El 23 de marzo de 2010, el presidente de los Estados Unidos Barack Obama promulgó la Ley de Protección al Paciente y Cuidado de Salud Asequible (Patient Protection and Affordable Care Act (PPACA)), que tiene como propósito reducir potencialmente el número de migrantes méxico-americanos sin cobertura médica (77). Con esta legislación los migrantes documentados y los hijos de migrantes nacidos en Estados Unidos: a) deberán de tener seguro de salud (exigencia llamada comúnmente "mandato individual"); b) serán elegibles para adquirir un seguro mediante créditos fiscales, con el fin de subsidiar el pago de dicho seguro; y c) se verán beneficiados por el incremento de la elegibilidad de Medicaid después de los cinco años de restricción, el cual incluirá a personas que ganen hasta el $133 \%$ de la línea de pobreza. Sin embargo, debido a que Medicaid es administrado por cada estado, estos pueden optar por no expandir dicho programa (78). No obstante que el número de personas protegidas por un seguro médico aumentará, los inmigrantes ilegales continuarán siendo elegibles para los programas otorgados por el Estado. Con la reforma, a ellos también se les prohíbe la compra de seguro de salud, incluso si pagan la totalidad del precio de su propio bolsillo (61). Es importante aclarar que los efectos reales de esta reforma se verán hasta que haya sido implementada, tanto federalmente como por los estados (79). En caso de que entre en vigor la PPACA, los migrantes legales se verán obligados a adquirir un seguro de gastos médico, abriendo la brecha entre los asegurados y los no asegurados, e imposibilitando a los migrantes indocumentados adquirir por cuenta propia un seguro médico. Esto obliga a los migrantes a obtener atención a la salud de forma privada o incluso fuerza su retorno.

Por otra parte, en el caso del acceso a la salud en México, como migrante no es posible acceder a la seguridad social, aunado a que esta es reducida, sobre todo en las zonas rurales con más índices migratorios, donde la falta de infraestructura afecta la debida provisión del servicio. Lo que deja como posibilidad la atención a la salud mediante la afiliación al Seguro Popular o mediante la atención privada, muchas veces inaccesible para las familias migrantes.

La atención privada se basa mayormente en la prestación directa de servicios en las unidades hospitalarias y consultorios privados, con fines de lucro (80), que además de ser muy grande no cuenta con una debida regulación por parte del gobierno federal o de los gobiernos locales (81). Existe una parte de la población que utiliza los servicios del seguro privado a través de seguros privados o pagos directos a prestadores de servicio privado de salud, lo cual la mayoría de las veces es costoso e inaccesible para toda la población. Se trata principalmente de población de clase media y alta; sin embargo, es importante señalar que existen personas con seguridad social o beneficiarias del Seguro Popular que se encuentran insatisfechos con los servicios a los cuales son beneficiarios o consideran que la calidad de estos o su cobertura no es suficiente y recurren al sector privado (66). Por ejemplo, la Ensanut 2006 señala que $25 \%$ de los derechohabientes de la seguridad social utilizan de manera regular los servicios privados de salud, sobre todo los servicios ambulatorios (aproximadamente diez millones y medio de derechohabientes). Es importante señalar que existen pocos estudios acerca de la atención privada de servicios de salud en México, a pesar del considerable y floreciente crecimiento de este sector. 


\section{Discusión}

Necesitamos más estudios que muestren el impacto de la legislación, los programas sociales y las instancias institucionales para los migrantes, tanto en el país de origen como en el de destino, los cuales como se demuestra a lo largo del cuerpo del presente trabajo se encuentran en una dinámica de doble exclusión.

Tal como se mencionó al inicio del texto, la mayor parte de la literatura se concentra en evidencia empírica a cada lado de la frontera, lo que limita la posibilidad de entender la dinámica en la cual se encuentran los migrantes. Cuando se utiliza la perspectiva transnacional se tienen en cuenta ambas comunidades, la de origen y la de destino, como si fuera un mismo espacio $(36-38,82)$. En el caso particular del presente trabajo, mediante este enfoque es posible tener una visión más completa de la dinámica de estos sujetos de derechos sociales y entender a qué pueden acceder los migrantes en este espacio, en donde se solapa la comunidad de destino y la de origen. Derechos que, por un lado, tienen altamente restringidos debido a su estatus legal y, por el otro, se encuentran limitados por su condición de migrantes y la falta de infraestructura del sistema de salud, creando lo que a lo largo del cuerpo del trabajo denominamos doble exclusión.

Con respecto al acceso a la salud en Estados Unidos, siendo migrante indocumentado o legal con una estancia menor a cinco años, es difícil ser elegible a algún programa de salud federal, lo cual puede variar dependiendo del estado en donde se resida, mientras que en México no es posible acceder a Seguridad Social (ISSSTE o IMSS) por el hecho de no contar con un trabajo formal en el país. Lo anterior deja al migrante con opciones muy limitadas. Por un lado, puede recurrir a seguros o consultas privadas, las cuales tienen costos generalmente inasequibles y, por otro lado, tiene la posibilidad de acudir a centros de beneficencia o caridad en Estados Unidos, los cuales muchas veces carecen de fondos, recursos humanos y posibilidades de colaboración con hospitales para casos de especialidad para atender a una población tan amplia como son los migrantes, o la afiliación al Seguro Popular en México, el cual tiene escaza cobertura y falta de infraestructura.

\section{Conclusiones: perspectivas en el futuro y reflexión acerca de la exclusión}

El caso de los mexicanos en los Estados Unidos muestra claramente cómo las problemáticas de atención a la salud se hacen aún más complejas al ser consideradas de manera transnacional. Sobre todo resalta que la población migrante vive en espacios donde la presencia del Estado para garantizar sus derechos sociales es bastante limitada, sin importar en qué lado de la frontera se encuentre. Lo anterior cuestiona la noción de que la migración es necesariamente una mejora para los individuos que escapan a las condiciones de marginación en los lugares de origen $(5,83-87)$. Asimismo, queda claro que la exclusión de los derechos de ciudadanía social no es simplemente un tema de falta de documentos en el lugar de destino, sino también de las limitantes estructurales de los países de origen. Por lo menos en el caso de la salud, los migrantes viven la inequidad también de manera transnacional $(88,89)$

Considerando todo lo anterior, es importante pensar en análisis académicos que deriven en políticas de salud no únicamente para un lado de la frontera, sino una verdadera salud transnacional, programas binacionales de salud permanentes (no temporales como los ya existentes), en donde se potencie la 
participación de las redes ya existentes, tales como los centros de beneficencia, los centros comunitarios y las iglesias, los cuales se adecuen a la realidad que viven los migrantes, en la cual se encuentran excluidos en ambos lados de la frontera, no únicamente en las comunidades de destino como se cree.

Además, el diseño de un sistema de control de enfermedades debe ir más allá del enfoque en la comunidad de destino. Por ejemplo, es importante la coordinación de los centros de salud en ambos países, sobre todo para el seguimiento a los tratamientos, actualizaciones y avances de los pacientes con padecimientos crónicos, los cuales son parte tanto de comunidades de origen como de comunidades de destino. Asimismo, es posible considerar esquemas de seguros de salud públicos o privados con cobertura binacional.

Estas alternativas son solamente algunas aristas que indican la importancia de pensar en los mecanismos de inclusión en los sistemas de salud desde los sujetos de la inclusión más que desde los mecanismos institucionales tradicionales del Estado. Es importante continuar insistiendo en la salud como un derecho fundamental, el cual debe ser garantizado con independencia del estatus migratorio de los mexicanos en Estados Unidos.

\section{Referencias bibliográficas}

1. Besserer F. Estudios transnacionales y ciudadanía transnacional. En: Mummert G, editor. Fronteras fragmentadas, identidades múltiples. Zamora: El Colegio de Michoacán; 1999. pp. 215-38.

2. Soysal Y. Limits to Citizenship: Migrants and Postnational Membership in Europe. Chicago: University of Chicago Press; 1994.

3. Ullmann SH, Goldman N, Massey DS. Healthier before they Migrate, Less Healthy when they Return? The Health of Returned Migrants in Mexico. Social Science \& Medicine. 2011; 73 (3): 421-8.
4. Castles S. Twenty-First-Century Migration as a Challenge to Sociology. Ethnic and Racial Studies. 2007 April 2007; 33 (3): 351-71.

5. De Haas H. Migration and Development. A Theoretica Perspective. International Migration Institute, Working Paper 9; 2008 [Internet] [acceso: 25 de abril del 2014]. Disponible en: http://www. imi.ox.ac.uk/.

6. Faist T, Fauser M, Kivisto P. The Migration Development Nexus. A Transnational Perspective. Basingstole: Palgrave McMillan; 2011.

7. Bedregal P, Zavala C, Atria J, Núñez G, Pinto MJ, Valdés S. Acceso a redes sociales y de salud de población en extrema pobreza. Revista Médica Chile. 2009; 137: 753-8.

8. Chavez LR. Doctors, Curanderos and Brujas: Health Care Delivery and Mexican Immigrants in San Diego. Medical Anthropology Quarterly. 1984; 15 (2): 31-7.

9. Gusmano MK. Undocumented Immigrants in the United States: U.S. Health Policy and Access to Care. Undocumented Patients Web Site. 2012 [Internet]. Disponible en: http://www.undocumentedpatients.org/issuebrief/health-policy-andaccess-to-care/.

10. Nigeda G, Ruiz-Larios JA, Bejarano-Arias RM, Alcalde-Rabanam JE, Bonilla-Fernandez P. Análisis de las alternativas de los migrantes mexicanos en Estados Unidos de América para atender sus problemas de salud. Salud Pública Mex. 2009; 51: 407-16.

11. Skaer TL, Robinso LM, Sclar DA, Harding GH. Utilization of Curanderos among Foreign Born Mexican-American Women Attending Migrant Health Clinics. Journal of Cultural Diversity. 1996; 3 (2).

12. Wallace SP, Torres J, Sadegh-Nobari T, Pourat N, Brown ER. Undocumented Immigrants and Health Care Reform. Los Angeles, California: UCLA Center for Health Policy Researcg; 2012.

13. Akincigil A, Sánchez Mayers R, Fulghum FH. Emergency Room Use by Undocumented Mexican Immigrants. Journal of Sociology \& Social Welfare. 2011; 38 (4): 33-50.

14. Caballero M, Leyva-Flores R, Ochoa-Marín SC, Zarco Á, Guerrero C. Las mujeres que se quedan: migración e implicación en los procesos de búsqueda de atención de servicios de salud. Salud Pública Mex. 2008; 5: 241-50.

15. Ransford HE, Carrillo FR, Rivera Y. Health Care-Seeking among Latino Immigrants: Blocked Access, Use of Traditional Medicine, and the Role of Religion. Journal of Health Care for the Poor and Underserved. 2010; 21 (3): 862-78.

16. Ramos ME. Similitudes y diferencias en la salud psicofísica entre mujeres y hombres migrantes en Estados Unidos. Implicaciones para las políticas de salud y programas de intervención social. En: UAM C, Departamento de Relaciones Sociales, editor. Mujer y migración, los costos emocionales. Mexico D. F.: UAM C; 2010.

17. Buttenheim A, Goldman N, Pebley AR, Wong R, Chung C. Do Meixcan immigrants "Import" Social 
Gradients in Health to the US? Social Science \& Medicine. 2010; 71 (7).

18. Connor A, Layne L, Thomisse K. Providing Care for Migrant Farm Worker Families in ther Unique Sociocultural Context and Environment. Journal of Transcultural Nursing Society. 2010; 21: 159-66.

19. Connor A, Rainer LP, Simcox JB, Thomisse K. Increasing the Delivery of Health Care Services to Migrant Farm Worker Families throough a Community Partnership Model. Public Health Nursing. 2007; 24 (4): 355-60.

20. Early J, Davis SW, Quandt SA, Rao P, Snively BM, Arcury TA. Housing Characteristics of Farmworker Families in North Carolina. Journal of Immigrant Minority Health. 2006; 8 (2): 173-84.

21. Stallones L, Vela Acosta MS, Sample P, Bigelow P, Rosales M. Perspectives on Safety and Health among Migrant and Seasonal Farmworkers in the United States and Mexico: A Qualitative Field Study. Journal of Rural Health. 2009; 25 (2): 219-25.

22. Vallejos QM, Quandt SA, Grzywacz JG, Isom S, Chen H, Galván L et ál. Migrant Farmworkers' Housing Conditions across an Agricultural Season in North Carolina. American Journal of Industrial Medicine. 2011; 54: 533-44.

23. Weigel MM, Armijos RX, Posada Hall Y, Ramírez Y, Orozco R. The Household Food Insecurity and Health Outcomes of U.S.-Mexico Border Migrant Seasonal Farmworkers. Journal of Immigrant Minority Health. 2007; 9: 157-69.

24. Gonzalez-Block MA, de la Sierra-de la Vega LA. Hospital Utilization by Mexican Migrans REturning to Mexico due to Health Needs. BMC Public Health. 2011; 11 (241).

25. Santos-Padrón H, Martínez-Calvo S, MartínezLópez MC, Álvarez-Malpica IL. La demanda potencial y la oferta de servicios de salud para las familias en México. Revista Gerencia y Políticas de Salud. 2011; 10 (21): 33-47.

26. Medina-Pasos AJ, Chel-Guerrero LA, NietoMontenegro S, Betancur-Ancona DA, Cason KL, Castellanos-Ruelas AF. Evaluación del estado de salud y nutrición de un grupo de emigrantes de retorno a Yucatán, México. Nutrición Clínica y Dietética Hospitalaria. 2010; 30 (1): 26-34

27. Hirsch JS, Higgins J, Bentley ME, Nathanson CA. The Social Constructions of Sexuality: Marital Infidelity and Sexually Transmitted Disease-HIV Risk in a Mexican Migrant Community. American Journal of Public Health. 2002; 92 (8): 1227-37.

28. Sánchez MA, Lemp GF, Magis-Rodríguez C, Bravo-García E, Carter S, Ruiz JD. The Epidemiology of HIV Among Mexican Migrants and Recent Immigrants in California and Mexico. Journal of Acquired Immune Deficiency Syndromes. 2004; 37: 204-14.

29. Magis-Rodríguez C, Lemp GF, Hernández M, Bravo-García E, Estrada F. Going North: Mexican Migrants and their Vulnerability to HIV. Journal of Acquired Immune Deficiency Syndromes. 2009; 51: 21-5.

30. Martínez-Donate AP, Rangel MG, Hovell MF, Santibáñez J, Sipan CL, Izazola JA. HIV Infection in
Mobile Populations: The case of Mexican Migrants to the United States. Revista Panamericana de Salud Pública. 2005; 17 (1): 26-9.

31. Heymann J, Flores-Macias F, Hayes JA, Kennedy M, Lahaie C, Earle A. The Impact of Migrtion on the Well-Being of Transnational Families: New Data from sending Communities in Mexico. Community, Work \& Family. 2009; 12 (1): 91-103.

32. Ochoa-Marín SC, Cristancho-Marulanda S, González-López JR. Mujeres compañeras de migrantes: imagen social y búsqueda de servicios de salud sexual y reproductiva. Revista Salud Pública. 2011; 13 (2): 183-95.

33. Frank R, Hummer RA. The Other Side of the Paradox: The Risk of Low Birth Weight among Infants of Migrant and Nonmigrant Households within Mexico. Center of Migration Studies of New York; 2002.

34. Hilderbrandt N, McKenzie DJ. The Effects of Migration in Child Health in Mexico. World Bank Policy Research Working Paper; 2005.

35. Schmeer K. Father Absence due to Migration and Child Illness in Rural Mexico. Social Science \& Medicine. 2009; 69 (8).

36. Levitt P. The Transnational Villagers. Berkeley: University of California Press; 2001.

37. Levitt P. Transnational Migration: Taking Stock and Future Directions. Global Networks. 2001; 1 (3): 195-216.

38. Mouw T, Chávez S, Edelblute H, Verdert A. Binational Social Networks and Assimilation: A test of the Importance of Transnationalism. Social Problems. 2004; 61 (3): 324-59.

39. Besserer F, editor. A Space of View: Transnational Spaces and Perspectives. Transnationalism: An Exchange of Theoretical Perspectives from Latina American, Africanist and Asian Anthropology; 1998; University of Manchester, UK: Manuscript.

40. Boehm DA. Intimate Migrations: Gender, Family, and Illegality among Transnational Mexicans. Nueva York: New York University Press; 2012.

41. Faist T. The Volume and Dynamics of International Migration and Transnational Social Spaces. Oxford: Clarendon Press; 2000. [xvi], 380 p.

42. Gasca Zamora J. Espacios transnacionales. Interacción, integración y fragmentación en la frontera México-Estados Unidos. México: Porrúa; 2002.

43. Glick Schiller N, Basch L, Blanc-Szanton C. Transnationalism: A New Analytical Framework for Understanding Migration. Annals of the New York Academy of Science. 1992; 645: 1-24.

44. Moctezuma Longoria M. Morfología y desarrollo de las asociaciones de migrantes mexicanos en Estados Unidos. Un sujeto social y político extraterritorial. Migración y Desarrollo. 2005; (5): 59-85.

45. Portes A, Escobar C, Walton A. Immigrant Transnational Organizations and Development: A Comparative Study. The International Migration Review. 2007; 4 (1): 242-81.

46. Castles S, Delgado Wise R. Migración y desarrollo: perspectivas desde el sur México: Porrúa; 2007. 
47. Delgado Wise R. Diez años de promover una agenda alternativa de investigación y acción. Migración y Desarrollo. 2013; 11 (20): 3-7.

48. Delgado Wise R, Covarrubias HM. Capitalist Restructuring, Development and Labour Migration: The Mexico-US Case. Third World Quarterly. 2008; 29 (7): 1359-74.

49. Faist T. Espacio social transnacional y desarrollo: Una exploración de la relación entre comunidad, Estado y mercado. Migración y Desarrollo. 2005; Segundo Semestre: 2-34.

50. Riosmena F, Massey DS. Pathways to El Norte: Origins, Destinations, and Characteristics of Mexican Migrants to the United States. International Migration Review. 2012 (1): 3.

51. Massey DS, Rugh JS, Pren KA. The Geography of Undocumented Mexican Migration. Mexican Studies = Estudios Mexicanos. 2010; 26 (1): 129-52.

52. Consejo Nacional de Evaluación de la Política de Dearrollo Social (Coneval). Metodología para la medición multidimensional de la pobreza en México. México, D. F: Coneval; 2010.

53. Singer LE. Leveraging Tax Exempt Status of Hospitals. Journal of Legal Medicine. 2008; 29 (7): 1350-5.

54. Light DW. Categorical Inequality, Institutional Ambivalence, and Permanently Failing Institutions: The Case of Immigrants and Barriers to Health Care in America. Ethnic and Racial Studies. 2011; 35 (1): 23-39.

55. Fortuny K, Chaudry A. A Comprehensive Review of Immigrant Access to Health and Human Services. Urban Institute; 2011.

56. Warner DC. Access to Health Services for Immigrants in the USA: From the Great Socity to the 2010 Health Reform Act and after. Ethnic and Racial Studies. 2012; 35 (1): 40-55.

57. Calvo JM. The Consequences of Restricted Health Care Access for Immigrants: Lessons from Medicaid and schip. Annals of Health Law. 2008; 17 (2): $175-212$.

58. Vargas Bustamante A, Fang H, Garza J, CarterPorkas O, Wallace SP, Rizzo JA et ál. Variations in Healthcare Access and Utilization among Mexican Immigrants: The Role of Documentation Status. Journal Immigrant Minority Healt. 2010; 14: 146-155.

59. Broder T, Blazer J. Overview of Immigrant Eligibility for Federal Programs. National Immigration Law Center; 2010.

60. Kaushal N, Kaestner R. Welfare Reform and Health Insurance of Immigrants. Health Services Research. 2005; 40 (3): 697-722.

61. Dordeski MV, Steffens KN. Immigrants and Healthcare: A Voice for Coverage. The Health Lawyer. 2010; 23 (2): 35-43.

62. Social Security Administration. Benefits for Children. Social Security Administration; 2012.

63. Laurell AC. Los seguros de salud mexicanos: cobertura universal incierta. Ciência \& Saúde Coletiva. 2011; 16 (6): 2795-806.

64. Cámara de Diputados. La seguridad social en México. México: Cámara de los Diputados; 2005.
65. Gabarrot Arenas M. Algunas Contradicciones en la política social mexicana. Bienestar y combate a la pobreza. Trace; 2013; 62: 36-49.

66. Frenk J, Gómez Dantés O. El sistema de salud en México. México: Ediciones N; 2008.

67. Martínez Narváez G. Un sistema en busca de salud: Desarrollo, declive y renovación del sistema de salud mexicano. México, D. F: Fondo de Cultura Económica; 2013.

68. Levy Algazy S. Imss-Oportunidades. En: La experiencia mexicana en salud pública: oportunidad y rumbo para el tercer milenio. México: Fondo de Cultura Económica; 2006. pp. 469-78.

69. Moctezuma Barragán G. Legislación y normalización. En: La experiencia mexicana en salud pública: oportunidad y rumbo para el tercer milenio. México: Fondo de Cultura Económica; 2006. pp. 567-76.

70. Urbina Fuentes M, Moguel Ancheita A, Muñiz Martelón ME, Solís Urdaibay JA. La experiencia mexicana en salud pública. México: Fondo de Cultura Económica; 2006.

71. Seguro Popular. Seguro Popular; 2012 [Internet] [acceso: 27 de mayo del 2014]. Disponible en: http:// www.seguro-popular.salud.gob.mx/.

72. Gonzalez-Block MA, Becker-Dreps S, de la Sierrade la Vega LA, González-Robledo LM, Olivares JC, York P et ál. Salud migrante, propuesta de un seguro binacional de salud. Cuernavaca, Morelos: Instituto Nacional de Salud Pública; 2008.

73. Durán-Arenas L, Salinas-Escudero G, GranadosGarcía V, Martínez-Valverde S. La transformación del sistema de salud con base en la atención primaria. Gaceta Médica de México. 2012; 148: 552-7.

74. Abrantes Pêgo R. La reforma del sector salud en México y la deuda pendiente con los desasistidos. Revista Gerencia y Políticas de Salud. 2011; 10 (21): 5-8.

75. Vizcarra-Bordi I, Farfán-González F. El programa "Vete Sano y Regresa Sano": Lecciones de una etnografía institucional en el Estado de México. Ruris. 2011; 6 (2): 123-53.

76. Farfán-González F, Vizcarra-Bordi I, González N. Políticas de salud. Estrategia biopolítica para controlar a los migrantes mexicanos en Estados Unidos. Migración y Desarrollo. 2012; 10 (19): 3-32.

77. Zuckerman S, Waidmann TA, Lawton E. Undocumented Immigrants, Left ouf of Health Reform, Likely to continue to Grow as Share of the Uninsured. Health Affairs. 2011; 30 (10): 1997-2004.

78. Vargas Bustamante A, Laugesen M, Caban M, Rosenau P. United States -Mexico Cross-Border Health Insurance Initiativs: Salud Migrante and Medicare in Mexico. Revista Panamericana de Salud Pública. 2012; 31 (1): 74-80.

79. McMorrow S, Kenney GM, Coyer C. Addressing Coverage Challenges for Children under the Affordable Care Act. Urban Institute; 2011.

80. Zurita B, Ramirez T. Desempeño del sector privado de la salud en México. Caleidoscopio de la Salud. Funsalud; 2003.

81. Jaramillo Cardona MC. La descentralización de la salud en México: avances y retrocesos. Revista Gerencia y Políticas de Salud. 2007; 6 (13): 85-111. 
82. Levitt P, Glick Schiller N. Conceptualizing Simultaneity: A Transnational Social Field Perspective on Society. IMR. 2004; 38 (3): 1002-39.

83. Conway D, Cohen J. Consequences of Remmittances for Mexican Transnational Communities. Economic Geography. 1998; 74 (1): 26-44.

84. Durand J, Parrado EA, Massey DS. Migradollars and Development: A Reconsideration of the Mexican Case. Int Migr Rev. 1996 Sum; 30 (2): 423-44.

85. Massey DS. Economic Development and International Migration in Comparative Perspective. Population and Developement Review. 1988; 14 (3): 383-413.

86. Nyberg-Sorensen N, Van Hear N, EngbergPedersen P. The Migration-Developement Nexus
Evidence and Policy Options State-of-the-Art Overview. International Migration. 2002 special issue 2; 40 (5): 5-43.

87. Stark O, Lucas REB. Migration, Remmittances, and the Family. Economic Developement and Cultural Change. 1988; 36 (3): 465-81.

88. Canales AI. La migración en la reproducción de la sociedad global. Migración y Desarrollo. 2013; 12 (Segundo Semestre): 9-41.

89. Faist T. "Ahora todos somos transnacionales": Relevancia de la transnacionalidad para comprender inequidades Sociales. Migración y Desarrollo. 2013; 11 (20): 67-105. 